\title{
Left-ventricular hypertrophy in 18-month-old donor rat hearts was not associated with graft dysfunction in the early phase of reperfusion after cardiac transplantation-gene expression profiling
}

\author{
Sevil Korkmaz-Icöz • Deniz Akca • Shiliang Li • Sivakkanan Loganathan • Paige \\ Brlecic • Mihály Ruppert • Alex Ali Sayour • Andreas Simm • Maik Brune • Tamás \\ Radovits • Matthias Karck • Gábor Szabó
}

Received: 6 December 2019 / Accepted: 24 February 2021 / Published online: 19 April 2021

(C) The Author(s) 2021

\begin{abstract}
The use of hearts with left-ventricular (LV) hypertrophy (LVH) could offer an opportunity to extend the donor pool for cardiac transplantation. We assessed the effects of LVH in 18-month-old spontaneously hypertensive stroke-prone (SHRSP) donor rats and following transplantation. In donors, cardiac function and structural alterations were assessed. Then, the hearts were transplanted into young normotensive-rats. We evaluated LV graft function $1 \mathrm{~h}$ after transplantation. The myocardial expression of 92 genes involved in apoptosis, inflammation, and oxidative-stress was profiled using PCR-array. Compared to controls, SHRSP-
\end{abstract}

S. Korkmaz-Icöz $(\bowtie) \cdot$ D. Akca $\cdot$ S. Li $\cdot$ S. Loganathan ·

P. Brlecic · M. Ruppert · A. A. Sayour · M. Karck •

G. Szabó

Laboratory of Cardiac Surgery, Department of Cardiac Surgery, University Hospital Heidelberg, 69120 Heidelberg, Germany

e-mail: korkmaz@uni-heidelberg.de

\section{S. Loganathan · A. Simm • G. Szabó}

Department of Cardiac Surgery, University Hospital Halle (Saale), 06120 Halle, Germany

M. Ruppert - A. A. Sayour - T. Radovits

Heart and Vascular Center, Semmelweis University,

1122 Budapest, Hungary

\section{Brune}

Department of Medicine I and Clinical Chemistry, Heidelberg University Hospital, 69120 Heidelberg, Germany rats developed LVH, had increased LV systolic performance (slope of the end-diastolic pressure-volume (PV) relationship: $1.6 \pm 0.2$ vs $0.8 \pm 0.1 \mathrm{mmHg} / \mu \mathrm{l}, p<0.05)$ accompanied by diastolic dysfunction [prolonged time constant of LV pressure decay (Tau: $15.8 \pm 0.6$ vs 12.3 $\pm 0.5 \mathrm{~ms}$ ) and augmented diastolic stiffness (LV enddiastolic PV relationship: $0.103 \pm 0.012$ vs 0.045 $\pm 0.006 \mathrm{mmHg} / \mathrm{ml}), p<0.05]$. They presented ECG changes, myocardial fibrosis, and increased nitrotyrosine immunoreactivity and plasma troponin- $\mathrm{T}$ and creatine kinase-CM levels. After transplantation, even though the graft contractility was better in SHRSP rats compared to controls, the adverse impact of ischemia/reperfusion-injury on contractility was not altered $\left(\mathrm{E}_{\mathrm{es}}\right.$ ratio after versus before transplantation: $32 \%$ vs $29 \%, p>0.05)$. Whereas nitrotyrosine immunoreactivity was higher, myeloperoxidase-positive cell infiltration was decreased in the SHRSP+transplanted compared to control+transplanted. Among the tested genes, LVH was associated with altered expression of 38 genes in donors, while transplantation of these hearts resulted in the change of four genes. Alterations in 18-month-old donor hearts, as a consequence of hypertension and $\mathrm{LVH}$, were not associated with graft dysfunction in the early phase of reperfusion after transplantation.

Keywords Left-ventricular hypertrophy; · Heart transplantation; · Spontaneously hypertensive strokeprone rats; $\cdot$ Ageing; $\cdot$ Gene expression 


\section{Introduction}

Heart transplantation is the current curative treatment option for end-stage heart failure. However, the imbalance between waiting lists and surgery rates continuously increases due to higher demand with no increase in the supply of suitable organs. Therefore, adequate and optimal utilization of the donor pool is essential. Efforts have been made to expand donor acceptance criteria by using socalled "appropriate marginal" donors, who would be declined under conventional transplant guidelines. The concept of marginal donors consists of donors who are older, have hepatitis $\mathrm{C}$ virus-positivity, a history of alcoholism, diabetes mellitus, an ejection fraction $<45 \%$, or a donor/recipient weight ratio $<0.7$ [5]. Nevertheless, the most common criteria causing a donor heart to be rejected include cold ischemia $>4 \mathrm{~h}$, donor age $>55$ years, left ventricular (LV) hypertrophy $(\mathrm{LVH})>1.3 \mathrm{~cm}$, and LV ejection fraction $\leq 50 \%$.

One potential approach is the inclusion of hypertrophied donor hearts harvested in the compensatory phase of hypertrophy [8]. Currently, hearts with LVH are not included in donor selection guidelines. LVH is a maladaptive response of the heart to chronic pressure overload. The myocardial morphological and electrophysiological changes, occurring to overcome a pressure overload, can be divided into three stages: developing hypertrophy, compensatory hypertrophy, and overt heart failure [6]. As a brief summary, we can declare that short-term adaptive cardiac hypertrophy becomes maladaptive in the long-term and may lead to further cardiac damage. Aortic valve stenosis, systemic hypertension, and artherosclerosis are clinical conditions associated with pressure-overload [21]. Systemic hypertension is defined as a repeatedly elevated blood pressure with a systolic pressure above $140 \mathrm{mmHg}$ and/or a diastolic pressure higher than $90 \mathrm{mmHg}$ [30]. It should be noted that more than one third of the population is affected in developed societies; therefore, patients with hypertensive cardiomyopathy could demonstrate a notable donor pool for heart transplantation [7]. Furthermore, the prevalence of LVH and abnormal LV geometry increases with age $[14,15]$. However, only sporadic data exists regarding the use of donor hearts with $\mathrm{LVH}$ in cardiac transplantation [8, 19] and experimental investigations evaluating the impact of LVH on both post-transplant graft function and molecular screening are still limited to date. Goland et al. have demonstrated that selected hearts from donors with mild and moderate LVH can be safely used for heart transplantation and may increase the number of hearts available for transplantation [8]. Wever et al. have showed that the use of allografts with LVH in association with other high-risk characteristics may result in increased mortality [31].

The stroke-prone spontaneously hypertensive (SHRSP) rat is a sub-type of spontaneously hypertensive (shr) that shows severe hypertension with high incidence of cerebral stroke attacks [23]. These rats are hypertensive at 5 weeks of age, and the hearts of 6- to 12-month-old spontaneously hypertensive rats have been reported to have wellcompensated LVH $[9,29]$. To investigate the effects of both ageing and $\mathrm{LVH}$, hearts from 18-month-old rats were used in the present study.

Taking this into consideration, we assessed the effects of LVH in 18-month-old SHRSP donor rats and following transplantation. In order to further identify molecular alterations occurring in the LVH donor heart before and after transplantation, gene expression changes associated with inflammation, apoptosis, and oxidative stress were investigated.

\section{Materials and methods}

\section{Animals}

Male SHRSP rats (Charles River, Sulzfeld, Germany) and age- and sex-matched normotensive Wistar rats were housed in a room at $22 \pm 2^{\circ} \mathrm{C}$ under 12 -h light/dark cycles, and were fed a standard rodent regime with water ad libitum. The final experiments on the rats were performed at an age of 18 months. The animals received humane care in compliance with the "Principles of Laboratory Animal Care" formulated by the National Society for Medical Research, and with the "Guide for the Care and Use of Laboratory Animals," prepared by the Institute of Laboratory Animal Resources and published by the National Institutes of Health (NIH Publication No. 86-23, revised 1996). This study was approved by the appropriate institutional review committees (G128/15). 
Normotensive and spontaneously hypertensive stroke-prone donors

\section{Experimental groups}

The rats were divided into two groups: (a) control donor rats and (b) SHRSP donor rats ( $n=6-8$ rats/group).

\section{Echocardiography}

One day prior to the final experiment, the rats were slightly anesthetized with $1.5-2.0 \%$ isoflurane by mask, the left side of the chest was shaved to obtain a clear image, and the animals were situated in the supine position on a warming pad. Transthoracic echocardiography was performed by using an HDI $5000 \mathrm{CV}$ echocardiography machine (ATL Ultrasound, Philips, Bothell, WA, USA) equipped with a $10-\mathrm{MHz}$ linear probe. Two-dimensional parasternal short-axis images as well M-mode recordings at the mid-papillary muscle level were assessed. The following parameters were measured: left-ventricular (LV) internal enddiastolic dimensions during diastole. LV mass was calculated to estimate the myocardial weight using the Devereux formula: LV mass $(\mathrm{g})=\{[$ (LVEDD $\left.\left.\left.+\mathrm{AWT}_{\mathrm{d}}+\mathrm{PWT}_{\mathrm{d}}\right)^{3}-\mathrm{LVEDD}^{3}\right] \times 1.04\right\} \times 0.8+0.14$ [4]. To exclude an influence of body weight differences, these parameters were normalized to body weight. In addition, LV volumes were estimated according to the Prolate method: LVEDV= $\left[(3.14 / 6) \times\right.$ LVEDD $\left.^{2}\right] \times \mathrm{L}$ [25]. The quantitative analysis of the LV systolic function consists of fractional shortening (FS), calculated as [(LVEDD-LVESD)/LVEDD] $\times 100, \mathrm{LV}$ ejection fraction, and cardiac output/index.

\section{Electrocardiography}

Before the hemodynamic measurements, the rats were anesthetized with sodium pentobarbital (60 $\mathrm{mg} / \mathrm{kg}$, i.p.) and kept in a supine position on heating pads, maintaining their core temperature (measured via a rectal probe) at $37^{\circ} \mathrm{C}$. As previously reported [11, 13], standard 12-lead electrocardiograms were recorded using subcutaneously placed needle electrodes.
In vivo left-ventricular cardiac function: pressure volume analysis

After the ECG recordings, as previously reported [11, 13], the rats were tracheotomised, intubated, and artificially ventilated with ambient air. A polyethylene catheter was inserted into the left external jugular vein for fluid administration. A 2F-microtip pressure-volume catheter was inserted into the right carotid artery and advanced into the ascending aorta. After a 5-min stabilization period, the arterial blood pressure was recorded, and the catheter was advanced into the left-ventricle under pressure control. With the use of a special pressure-volume-analysis program (PVAN, Millar Instruments, Houston, TX, USA), heart rate, systolic (SBP) and diastolic blood (DBP) pressures, mean arterial pressure (MAP), maximal slope of systolic pressure increment $\left(\mathrm{dP} / \mathrm{dt}_{\max }\right)$ and diastolic pressure decrement $\left(\mathrm{dP} / \mathrm{dt}_{\min }\right)$, and time constant of the LV pressure decay (Tau-g; according to the Glantz method and Tau-w by Weiss method [24]) were calculated. LV pressurevolume relations were assessed by transiently compressing the inferior vena cava. The slope of the LV end-systolic pressure-volume relationship (ESPVR) was calculated according to the linear $\left(E_{e s}\right)$ and the parabolic curviliniear model $\left(E_{\max }\right)$ [10]. The slope of the LV end-diastolic pressurevolume relationship (EDPVR) was calculated as a reliable index of LV stiffness.

\section{Biochemical analysis}

After hemodynamic measurements were completed, blood samples from the abdominal aorta were collected. After centrifugation $\left(4,500 \mathrm{~g}, 15 \mathrm{~min}, 4^{\circ} \mathrm{C}\right)$, plasma samples were obtained. The levels of high-sensitive cardiac troponin- $\mathrm{T}$ and creatine kinase-MB were determined in the central laboratory of the Heidelberg University clinic with ECLIA on Cobas E411 (Roche Diagnostics).

\section{Histopathology}

After the blood sample collection, all rats were sacrificed by bleeding and the hearts were explanted. Pieces of the LV myocardial tissue were fixed in buffered paraformaldehyde solution (4\%) and embedded in paraffin. Then, $5-\mu \mathrm{m}$ thick sections were stained with hematoxylin and eosin. Cardiomyocyte cross-sectional areas were calculated on a microscope using the $\mathrm{Cell}^{\wedge} \mathrm{A}$ 
software (Olympus Soft Imaging Solutions GmbH, Germany). An acid fuchsin orange G (AFOG)-stain was used to determine the extent of myocardial fibrosis. Four sections per heart were inspected under light microscopy and rated according to the following scoring system: grade 0 indicates normal tissue showing no fibrotic region; grade 1 indicates mild fibrosis; grade 2 indicates moderate fibrosis, and grade 3 indicates severe fibrosis. The histological evaluation was conducted by an analyst unaware of the experimental groups.

Rat model of heterotopic heart transplantation

\section{Experimental groups}

The rats were divided into two groups: (a) control+ transplanted group received hearts from normotensive donors and (b) SHRSP+transplanted group received hearts with LVH. The hearts was transplanted into young Sprague-Dawley rats ( $n=7-13$ rats per group).

\section{Surgical technique of heart transplantation}

Transplantations were performed from Wistar to Sprague-Dawley and SHRSP to Sprague-Dawley rat strains. The experimental model was described elsewhere $[13,16]$. Briefly, cardiac arrest was induced via Custodiol solution (Dr. Franz Köhler, Chemie GmbH, Bensheim, Germany), then the heart was explanted and immediately placed in Custodiol solution $\left(4^{\circ} \mathrm{C}\right)$. Recipient Sprague-Dawley rats were anaesthetized and then heparinized. The aorta and the pulmonary artery of the donor heart were anastomosed end to side to the abdominal aorta and the vena cava of the recipient rat, respectively. To minimize variability between surgical experiments, the duration between explantation and reperfusion was standardized to $1 \mathrm{~h}$. After completion of the anastomoses, the heart was reperfused with blood in situ for $1 \mathrm{~h}$.

\section{Functional measurement in the graft}

As previously reported [12], $1 \mathrm{~h}$ after transplantation a 3F latex balloon catheter (Edwards Lifesciences Corporation, Irvine, CA, USA) was introduced into the leftventricle via the apex and connected to a precisioncalibrated syringe for administration and withdrawal of fluid. Additionally, a Millar micromanometer (Millar Instruments, Houston, TX, USA) was inserted in the left-ventricle to determine LV systolic pressure, developed pressure, $\mathrm{dP} / \mathrm{dt}_{\max }$ and $\mathrm{dP} / \mathrm{dt}_{\min }$, LVEDP, and Tau at different $\mathrm{LV}$ volumes. $\mathrm{LV}$ volumes were calculated as the volume of saline injected into the balloon plus the volume of the empty balloon $(0.02 \mathrm{ml})$. Data for a complete pressure-volume curve were obtained through incremental increases in LV volume by $0.03 \mathrm{ml}$ until a volume of $0.17 \mathrm{ml}$ was reached.

Gene expression analysis

Using RT $^{2}$ Profiler ${ }^{\mathrm{TM}}$ PCR Array, the expression of 92 genes was profiled before and following heart transplantation. The official names of these genes are presented in (online Table 1). These genes were selected as they have been reported to be key genes involved in inflammation, apoptosis, and oxidative stress. Total RNA was extracted from LV myocardial samples with a miRNeasy Mini Kit (Qiagen, Hilden, Germany) and was reversetranscribed into cDNA using the $\mathrm{RT}^{2}$ First Strand Kit, mixed with $\mathrm{RT}^{2}$ qPCR Master Mix, containing SYBR Green, according to manufacturer's instructions (Qiagen, Hilden, Germany). In this Custom Array, the following non-regulated genes (genes-of interest) were used for normalization in the fold change expression data calculations: beta-2 microglobulin (B2m) and hypoxanthine-guanine phosphoribosyl transferase-1 (Hprt1). Genes with fold regulation greater than 2 or less than -2 at $p<0.05$ were considered as significantly altered.

Immunohistochemistry

LV myocardial tissue samples were fixed in buffered paraformaldehyde solution (4\%) and embedded in paraffin or stored in $-80^{\circ} \mathrm{C}$ until they could be cut into frozen sections. Then, blocks were cut into 5 - $\mu$ m-thick paraffin or frozen sections. The immunoreactivity to myeloperoxidase (MPO) (1:100; Abcam, Cambridge, UK) and nitrotyrosine (1:100; Abcam, Cambridge, UK) was investigated. Infiltrating neutrophils (MPOlabeled) were counted, and nitrotyrosine expression was semi-quantitatively assessed based on staining intensity and the distribution of the labelled target protein. Furthermore, we performed terminal deoxynucleotidyl transferase-mediated dUTP nick end-labeling (TUNEL) staining to detect DNA-strand breaks as described previously [13-15]. The number of TUNEL-positive cells was expressed as the ratio of DAPI-TUNEL double- 
Table 1 Body weight, heart weight, heart weight/body weight ratio, lung weight, lung weight/body weight ratio, cardiomyocyte diameter normalized to body weight, and myocardial collagen accumulation

\begin{tabular}{|c|c|c|c|}
\hline & Control & SHRSP & $p$ value \\
\hline Body weight $[\mathrm{g}]$ & $661 \pm 22$ & $322 \pm 4^{*}$ & $<0.0001$ \\
\hline Heart weight $[\mathrm{g}]$ & $2.03 \pm 0.10$ & $1.56 \pm 0.02 *$ & 0.0004 \\
\hline Heart weight / body weight $\times 1000$ & $3.09 \pm 0.14$ & $4.85 \pm 0.10^{*}$ & $<0.0001$ \\
\hline Lung weight $[\mathrm{g}]$ & $1.98 \pm 0.07$ & $1.43 \pm 0.06^{*}$ & $<0.0001$ \\
\hline Lung weight / body weight x1000 & $3.00 \pm 0.11$ & $4.45 \pm 0.17 *$ & $<0.0001$ \\
\hline Cardiomyocyte diameter / body weight $\left[\mu \mathrm{m}^{*} 1000 / \mathrm{g}\right]$ & $25.4 \pm 0.8$ & $67.7 \pm 2.4^{*}$ & $<0.0001$ \\
\hline Collagen accumulation (AFOG staining, score: $0-4$ ) & $0.78 \pm 0.1$ & $1.64 \pm 0.2 *$ & 0.0112 \\
\hline
\end{tabular}

SHRSP spontaneously hypertensive stroke-prone rats, $A F O G$ acid fuchsin orange G. $* P<0.05$ versus Control. $n=6-7$ rats/group

labeled nuclei to the total number of nuclei stained with 4', 6-diamidino-2-phenylindole (DAPI). Each specimen recieved an average score of four adjacent fields in a blinded fashion.

\section{Statistical analysis}

All data is expressed as mean \pm standard error of the mean (SEM). Statistical analyses of data were performed using GraphPad Prism 7.02 software (GraphPad Sofware, Inc., CA, USA). Before statistical tests were applied, the Shapiro-Wilk and D'Agostino-Pearson normality tests were used to assess normal distribution. For data with normal distribution, two-sample Student $t$-test was used to analyze the differences between the two groups. If the normality test failed, a nonparametric Mann-Whitney $U$-test was applied. In the case of PCR-Array gene expression, the $p$-values were calculated based on a Student's $t$-test of the replicate $2^{\wedge}$ (-Delta $\mathrm{Ct}$ ) values for each gene in the experimental groups. A value of $p<0.05$ was considered indicative of statistical significance.

\section{Results}

Detailed characterization of experimental model of LVH in 18-month-old donors

Body weight, heart and lung weights, cardiomyocyte diameter, and myocardial collagen accumulation

Compared to controls, SHRSP rats develop LVH, which was confirmed by significantly increased heart weight-to-body weight ratio, increased cardiomyocyte diameter normalized to body weight, and increased LV mass index. Additionally, excessive myocardial collagen accumulation in SHRSP rats indicated myocardial fibrosis. Furthermore, a decreased body weight, heart weight and lung weight, and an increase in lung weight-to-body weight was observed in the SHRSP rats compared to controls (Table 1, Figs. 1A-B and 2).

Neutrophil infiltration, nitro-oxidative stress, and DNA strand breaks

Immunoreactivity for nitrotyrosine was significantly higher in the SHRSP group compared to controls (score: $4.50 \pm 0.50$ versus $3.25 \pm 0.56, p<0.05)$. However, the number of MPO-positive cell infiltration ( $60 \pm 4$ versus $64 \pm 4$ positive cells, $p>0.05$ ) and the number of TUNELpositive nuclei ( $70 \pm 5$ versus $65 \pm 4 \%, p>0.05$ ) were not was significantly altered between SHRSP and control groups (Fig. 1C-E).

\section{Echocardiographic parameters}

Compared to controls, echocardiographic parameters displayed significantly increased stroke volume index, ejection fraction, and cardiac index in SHRSP rats (Table 2, Figs. 2A and 3).

\section{Hemodynamic parameters}

Compared to controls, SHRSP rats had increased LV systolic performance (including indices of load-dependent (maximum rate of rise of leftventricular pressure $\mathrm{dP} / \mathrm{dt}_{\max }$ and cardiac index, ejection fraction) and enhanced load-independent 


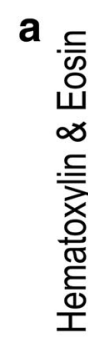

b
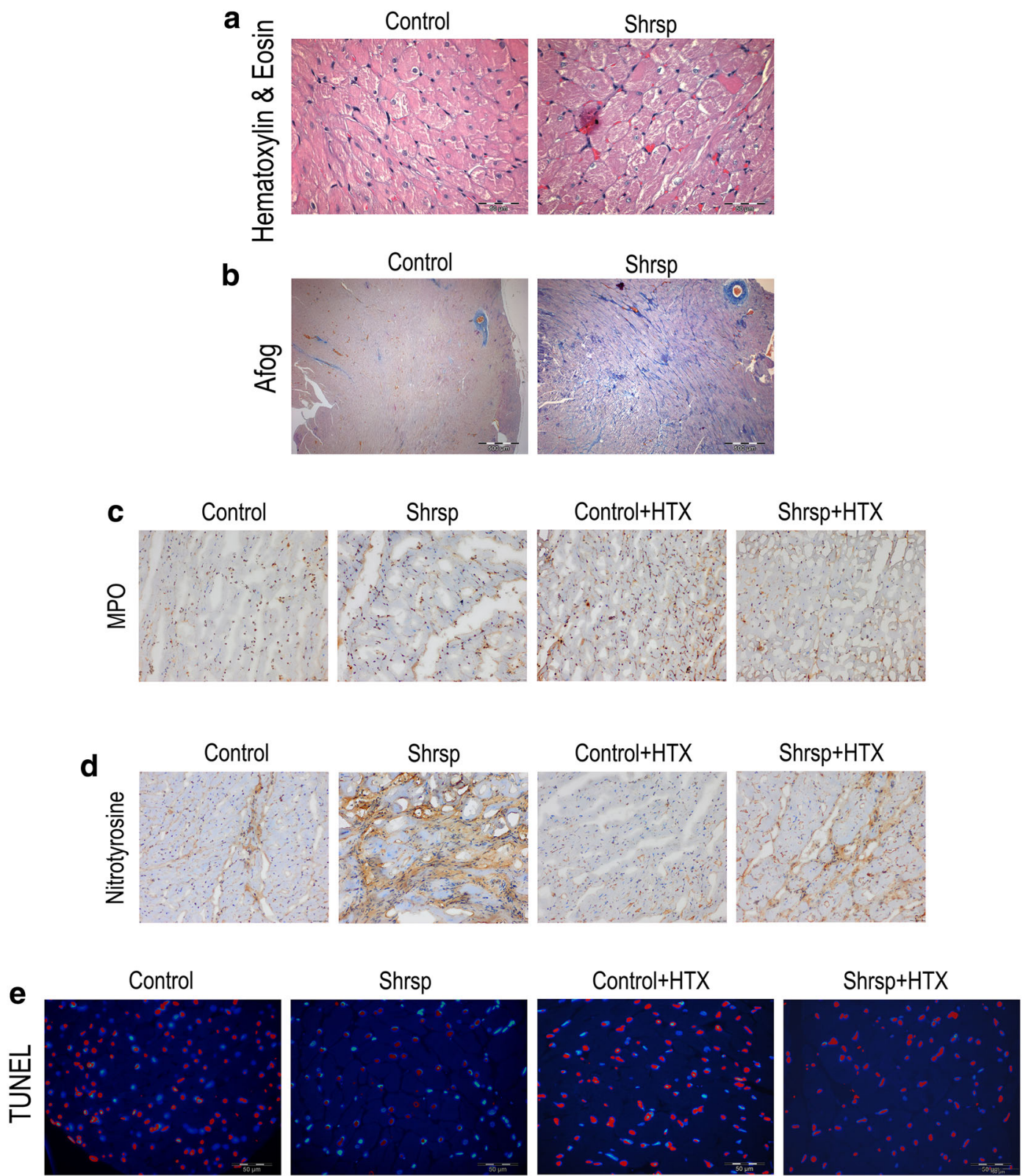

Fig. 1 Representative photomicrographs of myocardium in donors and after transplantation. (A) hematoxylin and eosin $(\times 400$, scale $50 \mu \mathrm{m}),(\mathrm{B})$ acid fuchsin orange $\mathrm{G}$ (Afog) $(\times 100$, sclae 500 $\mu \mathrm{m}),(\mathrm{C})$ myeloperoxidase $(\mathrm{MPO})(\times 200$, scale $50 \mu \mathrm{m}),(\mathrm{D})$ nitrotyrosine $(\times 200$, scale $50 \mu \mathrm{m})$, and (E) terminal

deoxynucleotidyl transferase-mediated dUTP nick end-labeling (TUNEL) $(\times 400$, scale: $50 \mu \mathrm{m})$ in control and SHRSP rats. SHRSP indicates stroke-prone spontaneously hypertensive rats and HTX, heart transplantation

(the slopes $\mathrm{E}_{\mathrm{es}}$ and $\mathrm{E}_{\max }$ of the end-systolic PV relationship) parameters in the presence of diastolic dysfunction [(prolonged time constant of LV pressure decay Tau) and increased diastolic stiffness (slope of the end-diastolic PV relationship)] (Table 2, Figs. 2B and 3).

\section{Electrocardiogram patterns}

On ECG recording, compared to controls, SHRSP rats displayed a significant increase in QRS complex duration and prolongation of corrected QT (Table 2, Figs. 2C and 3). 

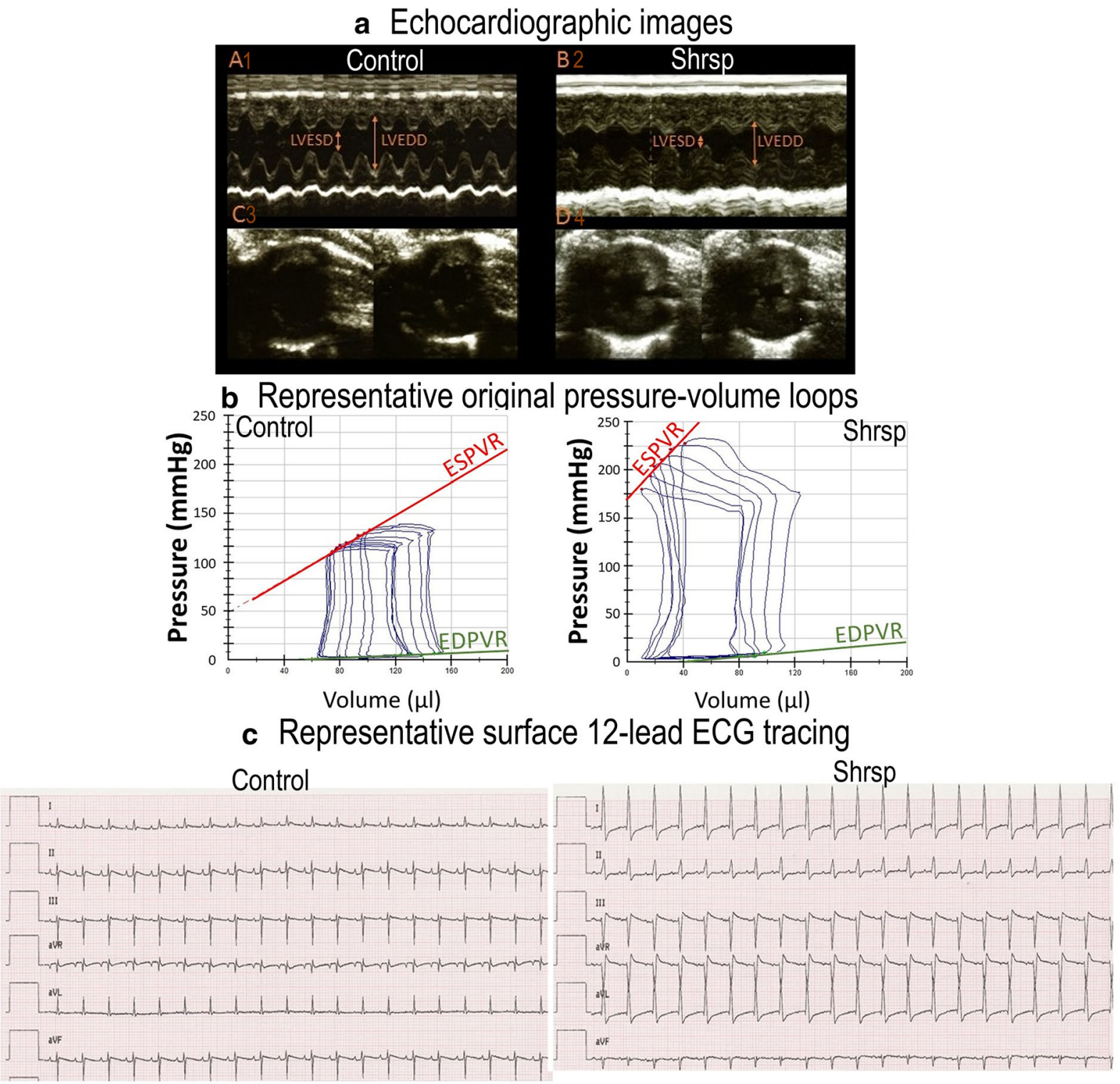

Fig. 2 Representative echocardiogram, pressure-volume loops, and surface electrocardiogram (ECG) in donors. (A) Representative two-dimensional echocardiographic images (A1-B1) Mmode recordings and (C1-D1) short axis in control and SHRSP rats. (B) Representative pressure-volume loops obtained with a micromanometer conductance catheter system at different preload. End-systolic pressure-volume relationship (ESPVR) and end-

\section{Biochemical parameters}

Plasma levels of cardiac Troponin-T $(401 \pm 107$ vs 89 $\pm 29 \mathrm{pg} / \mathrm{ml}, p=0.0173)$, and creatine kinase-MB $(370 \pm 60$ vs $166 \pm 16 \mathrm{U} / \mathrm{I}, p=0.006$ ) were significantly increased in SHRSP rats compared to the control group. diastolic pressure-volume relationship (EDPVR) in one representative animal in control and SHRSP groups. (C) Representative surface ECG tracings in control and SHRSP rats. SHRSP indicates stroke-prone spontaneously hypertensive rats; LVESD, leftventricular end-systolic dimension; LVEDD, left-ventricular enddiastolic dimension

Effect of LVH on graft function after transplantation

After heart transplantation, significantly increased LV systolic pressure, developed pressure, and $\mathrm{dP} / \mathrm{dt}_{\max }$ were observed in the SHRSP hearts when compared with the control group, indicating an increased systolic function 
Table 2 Echocardiographic parameters, in vivo left-ventricular cardiac function by pressure-volume analysis, and electrocardiographic parameters in donors

\begin{tabular}{|c|c|c|c|}
\hline & Control & SHRSP & $p$-value \\
\hline \multicolumn{4}{|l|}{ Echocardiographic parameters } \\
\hline Heart rate $[$ beats/min] & $342 \pm 16$ & $347 \pm 51$ & 0.81 \\
\hline LV end-diastolic diameter $[\mathrm{mm}]$ & $7.17 \pm 0.15$ & $5.51 \pm 0.15^{*}$ & $<0.0001$ \\
\hline Fractional shortening $[\%]$ & $38.0 \pm 1.7$ & $41.5 \pm 1.5$ & NS \\
\hline LV end-diastolic volume $[\mu \mathrm{l}]$ & $449 \pm 25$ & $264 \pm 15.5^{*}$ & $<0.0001$ \\
\hline LV end-systolic volume $[\mu \mathrm{l}]$ & $157 \pm 13$ & $75 \pm 3.9^{*}$ & $<0.0001$ \\
\hline Stroke volume $[\mu l]$ & $292 \pm 17$ & $189 \pm 14.4^{*}$ & $<0.0005$ \\
\hline Cardiac output $[\mu 1 / \mathrm{min}]$ & $100888 \pm 8656$ & $64962 \pm 3945^{*}$ & $<0.05$ \\
\hline \multicolumn{4}{|c|}{ In vivo LV cardiac function by pressure-volume analysis } \\
\hline Systolic blood pressure $[\mathrm{mmHg}]$ & $137 \pm 5$ & $221 \pm 7 *$ & $<0.0001$ \\
\hline Diastolic blood pressure $[\mathrm{mmHg}]$ & $111 \pm 4$ & $166 \pm 5^{*}$ & $<0.0001$ \\
\hline Mean arterial pressure $[\mathrm{mmHg}]$ & $120 \pm 4$ & $184 \pm 5^{*}$ & $<0.0001$ \\
\hline Heart rate [beats/min] & $369 \pm 9$ & $321 \pm 7^{*}$ & 0.0001 \\
\hline Tau-w [ms] & $10.7 \pm 0.6$ & $11.8 \pm 0.4$ & NS \\
\hline $\mathrm{E}_{\max }(\mathrm{ESPVR})[\mathrm{mmHg} / \mu \mathrm{l}]$ & $2.75 \pm 0.18$ & $5.92 \pm 0.62 *$ & $<0.001$ \\
\hline \multicolumn{4}{|l|}{ Electrocardiographic parameters } \\
\hline Heart rate [beats/min] & $342 \pm 16$ & $347 \pm 10$ & NS \\
\hline $\mathrm{RR}[\mathrm{ms}]$ & $178 \pm 9$ & $174 \pm 5$ & NS \\
\hline $\mathrm{PR}[\mathrm{ms}]$ & $43 \pm 2$ & $48 \pm 2$ & NS \\
\hline $\mathrm{QT}[\mathrm{ms}]$ & $58 \pm 3$ & $93 \pm 3^{*}$ & $<0.0001$ \\
\hline
\end{tabular}

$L V$ left-ventricular, Tau-w time constant of LV pressure decay (according to the Weiss method), ESPVR end-systolic pressure-volume relationship, $S H R S P$ spontaneously hypertensive stroke-prone rats. $* P<0.05$ versus control. $n=19-22$ rats/group

(Fig. 4A-C). Furthermore, $\mathrm{dP} / \mathrm{dt}_{\text {min }}$ was significantly higher and Tau-w was significantly decreased in the SHRSP group compared to controls (Fig. 4D-E). Even though graft contractility was better in SHRSP rats compared to controls, the adverse impact of ischemia/reperfusion injury on contractility was not altered $\left(E_{\mathrm{es}}\right.$ ratio after to before transplantation: $32 \%$ versus $29 \%, p>0.05$ ).

Effect of LVH on neutrophil infiltration, nitrotyrosine, and DNA-strand breaks after transplantation

Immunohistochemical analysis shows that LVH significantly decreased MPO-positive cell infiltration $(49 \pm 5$ versus $64 \pm 5$ positive cells, $p<0.05)$ and increased nitrotyrosine immunoreactivity (score: $3.46 \pm 0.49$ versus $2.13 \pm 0.26, p<0.05$ ) in the SHRSP+transplanted group compared to the control+transplanted rats (Fig. 1). After transplantation, DNA-fragmentation, as reflected by an increased number of TUNEL-positive nuclei was observed in the control+transplanted versus control $(75 \pm 4$ versus $65 \pm 4 \%, p<0.05)$ and in the SHRSP+ transplanted versus SHRSP ( $83 \pm 2$ versus $70 \pm 5 \%$, $\mathrm{p}<0.05$ ) (Fig. 1).

Changes in cardiac gene expression caused by LVH in donors and their implication in heart transplantation

SHRSP donor heart versus normotensive control donor heart

To determine the effects of LVH-induced cardiac changes, 92 genes in the donor heart were surveyed. Among them, the expression of 38 genes were significantly altered in SHRSP hearts compared to controls (2 genes were upregulated: Gpx1, Ccl20 and 36 genes down regulated: Akt1, Aox1, Apafl1, Bcl2l1, Birc3, Casp1, Casp2, Casp4, Casp8, Cat, Ccl24, Ccl3, Ccl4, Ccl5, Ccr2, Ccr3, Cd401g, Epx, Fas, Fasl, Il13, Il15, Il16, Il3, Il4, Il7, Il9, Mpo, Ncf1, Nos2, Noxo1, Tgfb1, Tnf, Tnfrsf10b, Tnfsf10, Tpo, Table 3, first column). 
Echocardiographic parameters
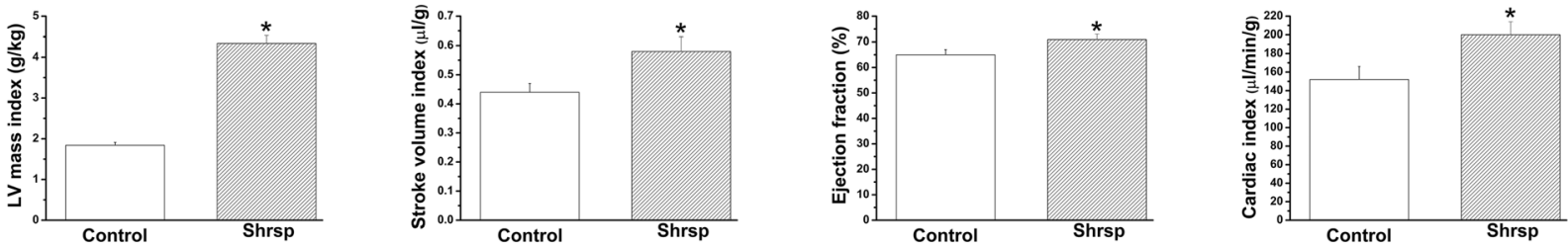

In vivo LV cardiac function by pressure-volume analysis
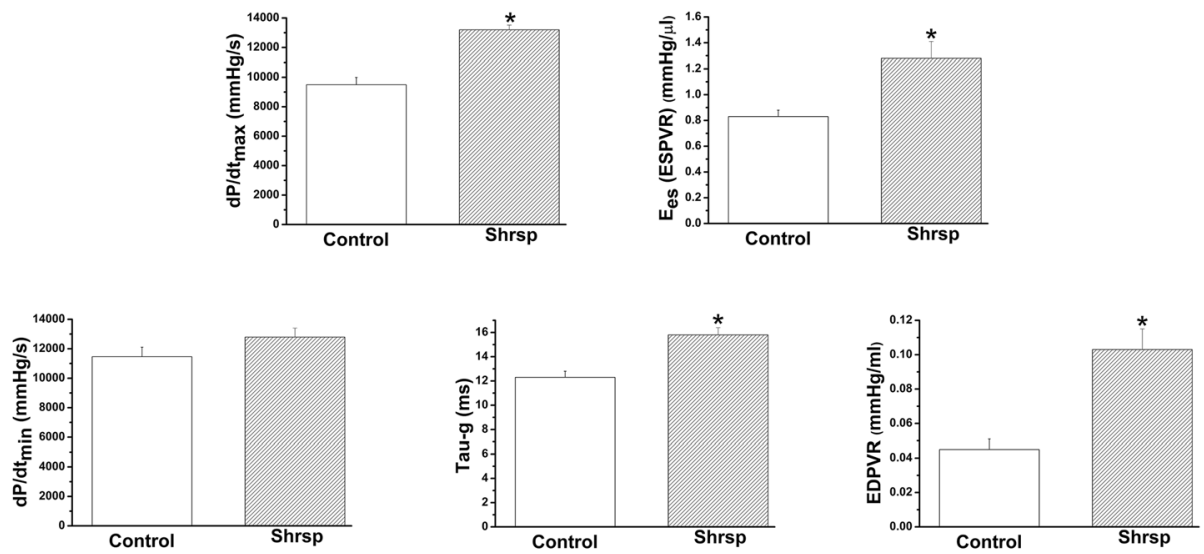

Electrocardiographic parameters

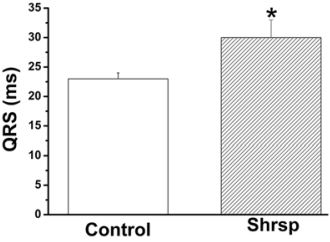

Fig. 3 Echocardiographic parameters, in vivo left-ventricular (LV) cardiac function by pressure-volume analysis, and electrocardiographic parameters in donors. SHRSP indicates spontaneously hypertensive stroke-prone rats; Tau-g, time constant of LV

\section{After transplantation versus before transplantation}

To determine the effects of heart transplantationinduced cardiac changes, 92 genes were surveyed. In normotensive hearts (control+transplanted versus control), among the tested genes, 18 genes were significantly altered compared to controls ( 10 genes were upregulated: Birc3, Ccl3, Fos, Hmoxl, Ill1, Il6, Nos2, Sele, Srxn1, Tnfrsfla and 8 genes downregulated: Cc12, Ccr2, Epx, Il15, Il16, Il7, Sod3, Tnfsf10, Table 3, second column). In LVH hearts (SHRSP+transplanted versus SHRSP), among the tested genes, 20 genes were

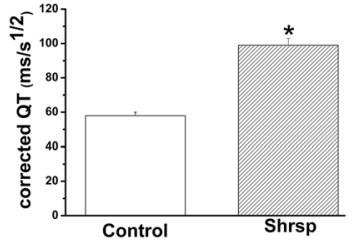

pressure decay (according to the Glantz method); ESPVR, endsystolic pressure-volume relationship; EDPVR, end-diastolic pressure-volume relationship. ${ }^{*} P<0.05$ versus Control. $n=19-22$ rats/group

significantly altered (13 genes were upregulated: Birc3, Ccl3, Ccl4, Fos, Hmox1, Il10, Il11, Il6, Jun, Nos2, Sele, Srxn1, Tnf and 7 genes downregulated: Ccr2, Epx, Il15, Il16, Il7, Serpinb1b, Tnfsf10, Table 3, third column). However, there are 15 common genes (Table 3, second and third columns); therefore, in SHRSP rats, transplantation significantly altered 5 additional genes (Jun, Ccl4, Il10, Tnf, Serpnb1, Table 3, third column). Ccl4 and Tnf, which were down regulated in SHRSP rats compared to normotensive rats (Table 3, first column), were significantly upregulated after transplantation (Table 3, third column). The three altered genes Tnfrsfla, Sod3, and 


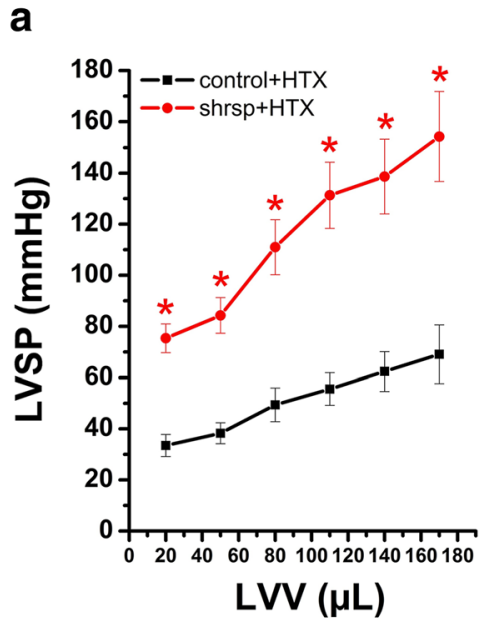

d

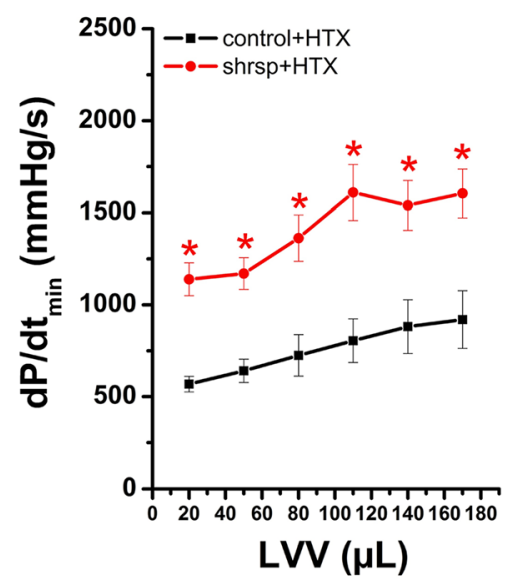

Fig. 4 In vivo left-ventricular (LV) graft function after heart transplantation (HTX). SHRSP indicates spontaneously hypertensive stroke-prone rats; LVSP, LV systolic pressure; $\mathrm{dP} / \mathrm{dt}_{\max }$, maximum rate of rise of left-ventricular pressure; $\mathrm{dP} / \mathrm{dt}_{\min }$,

Ccl12 observed in control+transplanted versus control hearts were not found in SHRSP+transplanted versus SHRSP groups.

\section{Graft with LVH versus control graft \\ after transplantation}

To determine the effects of both LVH and heart transplantation-induced cardiac changes, 92 genes were surveyed in the graft. The clustergrams create a heat map with dendrograms to indicate which genes are coregulated (Fig. 5). Among the tested genes, 34 genes were significantly altered in SHRSP+transplanted rats compared to control+transplanted group (3 genes were upregulated: Ccl11, Ccl20, Gpx1 and 31 genes

e
C

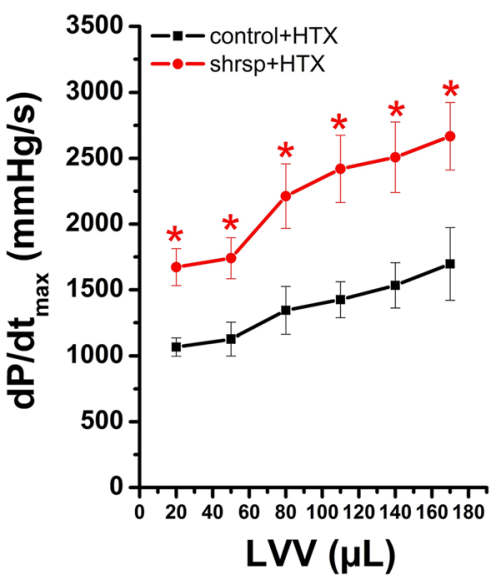

$\operatorname{LVV}(\mu \mathrm{L})$

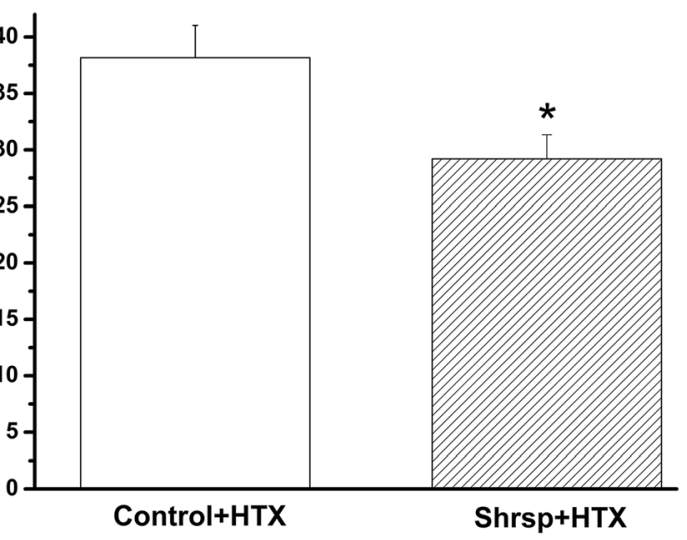

maximum rate of fall of left-ventricular pressure; LVEDP, LV end-diastolic pressure; Tau, time constant of LV pressure decay (at an intraventricular volume of $50 \mu \mathrm{l}$ ); and LVV, LV volume. $* P<0.05$ versus Control. $n=7-13$ rats/group

downregulated: Akt1, Aox1, Bcl2l1, Birc3, Casp1, Casp2, Casp8, Cat, Ccl24, Ccl5, Ccr2, Ccr3, Duox1, Epx, Fas, Faslg, Il13, Il15, Il16, Il3, Il4, Il7, Il9, Mpo, Ncf1, Nos2, Serpinb1b, Srxn1, Tnfrsf10b, Tnfsf10, Tpo, Table 3 fourth column). However, among these 34 genes, 30 genes were common with those of Table 3, first column, i.e., the alteration is due to $\mathrm{LVH}$ and not to the transplantation. In other word, the additive effect of LVH and transplantation caused the alteration of 4 genes (Ccl11, Duox1, Serpinb1b, and Srxn1, Table 3, fourth column). Furthermore, the altered expression of 8 genes due to LVH was normalized after transplantation in SHRSP rats, i.e., they are likely not involved in graft changes after transplantation. 


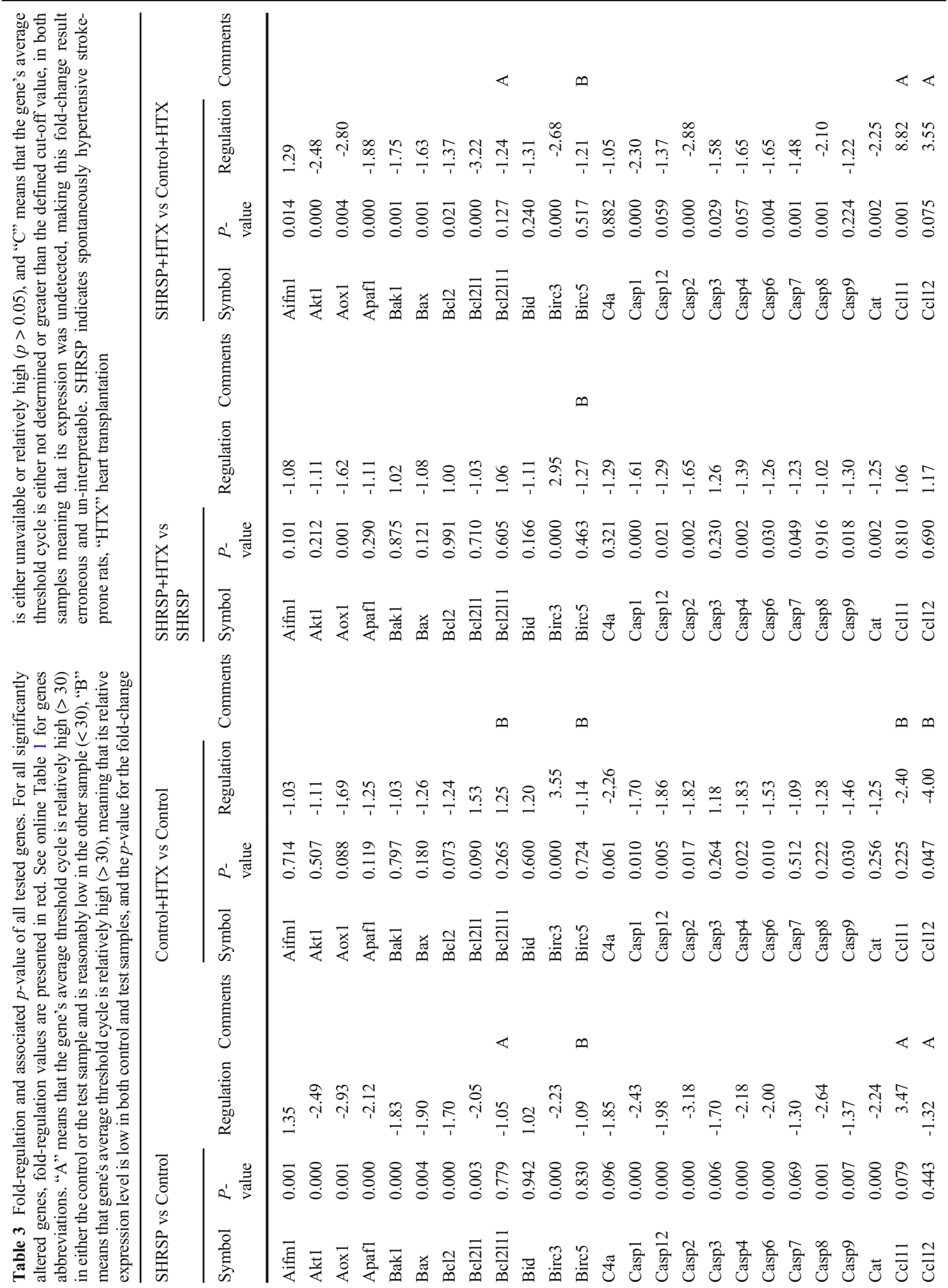




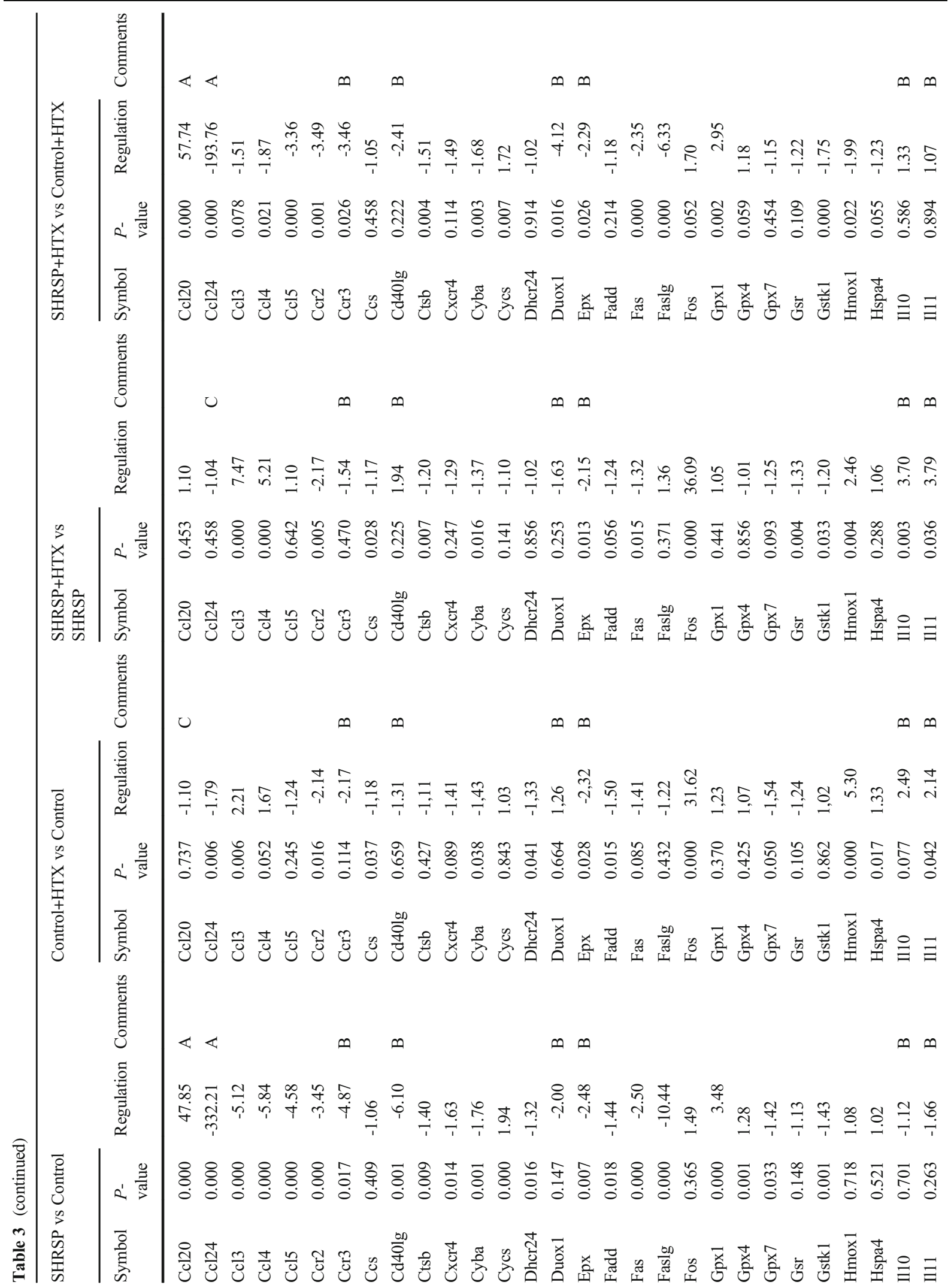




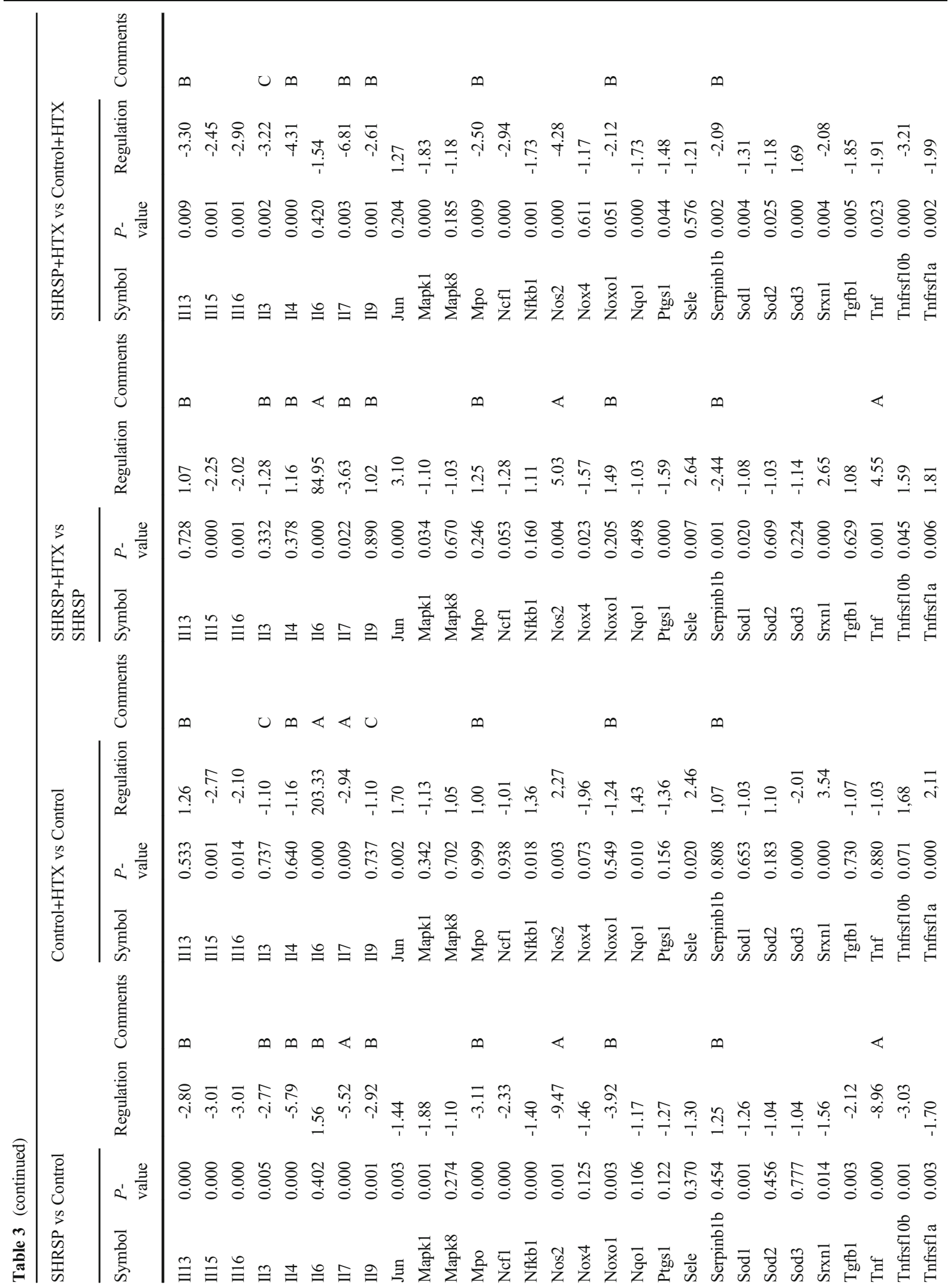


<smiles>C1=CC=CCC=CC=C1</smiles> 


\section{a Shrsp versus Control versus Control+HTX}

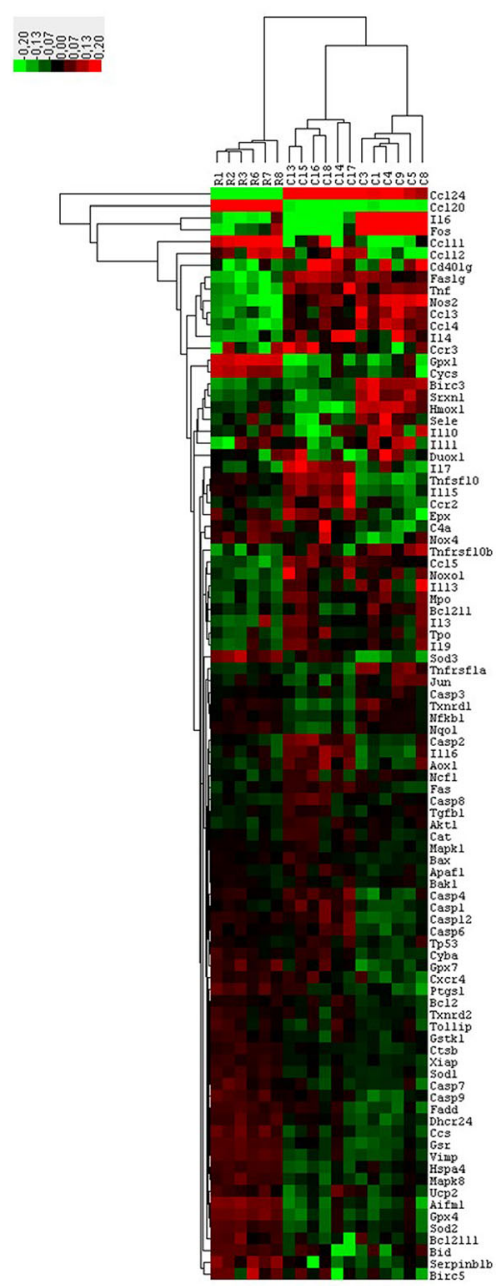

Fig. 5 Changes in cardiac gene expression caused by leftventricular hypertrophy in donors and their implication in heart transplantation. The expression of 92 genes involved in inflammation, apoptosis, and oxidative stress has been profiled in donors and in the graft after transplantation. Clustergrams create a heat map with dendograms to indicate which genes are co-regulated. Degrees of red and green indicate relatively high and low expression of the corresponding gene, respectively, and black squares denote genes equally expressed. (A) SHRSP versus Control and Control+HTX versus Control groups, (B) SHRSP+HTX versus

\section{Discussion}

In this study, we investigated the effect of LVH on early post-transplant changes at functional levels and analyzed gene expression profiles in the hearts of 18month-old SHRSP rats. To the best of our knowledge,
Shrsp versus Shrsp+HTX

\section{c Shrsp+HTX versus} Control+HTX

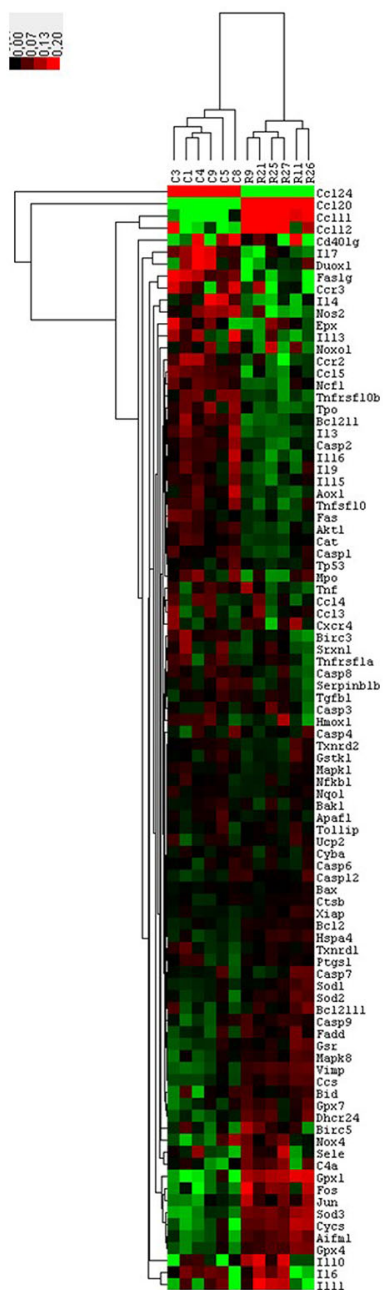

SHRSP groups, and (C) Shrps+HTX veruss Control+HTX groups. The $\mathrm{x}$-axis indicates the rat's number ["C" corresponds to Control group, including six rats $(\mathrm{C} 13, \mathrm{C} 14, \mathrm{C} 15, \mathrm{C} 16, \mathrm{C} 17$, and $\mathrm{C} 18)$, Control+HTX group, including six rats $(\mathrm{C} 1, \mathrm{C} 3, \mathrm{C} 4$, $\mathrm{C} 5, \mathrm{C} 8$, and C9), and " $\mathrm{R}$ " to SHRSP group, including six rats (R1, R2, R3, R6, R7, and R8), SHRSP+HTX group, including six rats (R9, R11, R21, R25, R26, and R27)], and the y-axis indicates the genes. SHRSP indicates spontaneously hypertensive stroke-prone rats, "HTX" heart transplantation. $n=6$ rats/group

this is the first report demonstrating that the alterations in donors, as consequence of hypertension and $\mathrm{LVH}$, were not associated with LV graft dysfunction in the early phase of reperfusion after transplantation. Changes in the expression of antioxidant enzyme genes (dual oxidase-1, serine peptidase inhibitor $b 1 b$, and 
sulfiredoxin-1), the inflammatory cytokine $\operatorname{ccl11}$, and reduced neutrophil infiltration were observed in the LVH hearts after transplantation. Taken together, decreased acute inflammatory response rather than reduced nitro-oxidative stress may, in part, explain the tolerance of donor hearts with LVH to ischemia/ reperfusion injury during heart transplantation.

Efforts have been made to extend the acceptance criteria for hearts used for transplantation to allow the use of organs from "marginal" donors. However, there is little data regarding the transplantation of donor hearts with $\mathrm{LVH}[1,8,19]$ and the pathomechanisms of potential heart grafts from donors with $\mathrm{LVH}$ in cardiac transplantation have not been completely elucidated. Therefore, we characterized the effects of LVH in potential donor rats. In a previous study, Cingolani et al have shown that at 10 to 11 months of age, SHR have increased systolic performance accompanied by delayed relaxation and increased diastolic stiffness, using a pressure-volume conductance catheter system [2]. Our in vivo results confirmed enhanced cardiac mass leading to increased LV contractility (due to the adequate hypertrophic response) in the presence of diastolic dysfunction (due to an increase in wall thickness/fibrosis and alterations in relaxation/myocardial stiffness) in 18-month-old SHRSP donor rats compared to age-matched controls. Furthermore, the release of intracellular cardiac enzymes or markers in the circulation, such as troponin- $\mathrm{T}$, lactate dehydrogenase, creatine kinase reflects cellular membrane damage and/or death of cardiomyocytes $[18,26]$. Severe hypertension can cause relative ischemia due to the higher energy demand of the hypertrophied myocardium that is not met by the coronary circulation even in the absence of occlusion. In our study, hypertrophied hearts released more plasma levels of cardiac troponin- $\mathrm{T}$ and creatine kinase MB than control hearts, indicating cardiomyocyte injury. Furthermore, LVH increased the immunoreactivity for nitrotyrosine, a nitrooxidative stress marker. Finally, we profiled the myocardial expression of 92 genes in these rats. To the best of our knowledge, this is the first report describing that LVH altered the expression of 38 genes, involved in apoptosis, oxidative stress, and inflammatory response, in donor hearts. This may indicate a relationship between these genes and the cause of LVH in 18-month-old SHRSP rats.

Myocardial ischemia/reperfusion injury during heart transplantation impairs early graft function, contributing to adverse short-term [17] and long-term [32] graft outcome in the recipients. For the success of heart transplantation, a fast functional recovery of the transplanted donor heart is essential and an important determinant of the long-term outcome [27]. As profound hemodynamic changes occur during the early phase after transplantation, we focused our investigations on the early phase of reperfusion. Our data demonstrated that alterations observed in donors with $\mathrm{LVH}$ were not associated with LV graft dysfunction after heart transplantation. Involvement of inflammatory mechanisms in fibrotic processes is one of the main components of ventricular remodeling process [22]. Additionally, it is well recognized that cardiomyocyte specific apoptosis contributes to the transition from LVH to LV dysfunction [3]. Furthermore, oxidative stress has been identified as one of the key contributing factors in the development of cardiac hypertrophy [20]. Taken together, we used mRNA expression profiling to identify myocardial gene expression changes related to inflammation, apoptosis, and oxidative stress in donors and after heart transplantation. We profiled the expression of 92 genes. We showed that alterations of 38 genes were related to LVH (SHRSP versus normotensive rats), because their gene expression changes were independent from the effect of transplantation. The gene expression of 18 genes was, however, affected only by transplantation, independent of LVH (normotensive+transplanted versus normotensive rats). Furthermore, in SHRSP rats, transplantation significantly altered 5 additional genes (jun, ccl4, Il10, tnf, serine peptidase inhibitor b1b) (SHRSP+transplanted versus SHRSP). The additive effect of both LVH and transplantation was enough to alter the expression of serine peptidase inhibitor $b 1 b$, dual oxidase-1, sulfiredoxin-1, and ccl11 (SHRSP+ transplanted versus normotensive+transplanted). Additionally, the expression of sulfiredoxin-1 was significantly up-regulated in the normotensive+transplanted group compared to normotensive group $(+3.54)$ and in the shrps+transplanted group compared to SHRSP group (+2.65). LVH showed no effect on its gene expression. Our results suggest that the alteration of sulfiredoxin-1 gene expression was the effect of transplantation alone. We did see a significant downregulation of sulfiredoxin-1 gene expression $(-2.08)$ in the SHRSP+transplanted group compared to normotensive+transplanted group. Although, at first glance, it seems that sulfiredoxin-1 expression was only affected following transplantation, and LVH had no effect on its expression, it appears that LVH had an invisible effect on the tested genes. This became evident after an additional second injury. Sulfiredoxin-1, an endogenous antioxidant, has been shown to protect against simulated 
ischemia/reperfusion injury in cardiomyocytes [33] that is associated with reactive oxygen species-mediated cell death. Additionally, our PCR array results showed that ccll1 expression was significantly increased $(+8.8)$ in the shrps+transplanted group compared to normotensive+ transplanted group. Zweifel et al. have shown that posttransplantation myocardial fibrosis correlated with eotaxin/ CCL11 levels in rat models of transplantation [34]. They suggested that targeting eotaxin/CCL11 with monoclonal antibodies could reduce cardiac mast cell infiltration, possibly resulting in decreased myocardial fibrosis and improved contractile function after heart transplantation [34]. Neutrophils, circulating leucocytes, are usually the earliest cells to infiltrate transplanted tissue and their recruitment/ activation plays an important role in transplant injury [28]. Our results show that whereas LVH has no effect on neutrophil infiltration in donor hearts, it decreases neutrophil count after transplantation. Surprisingly, we found that nitro-oxidative damage, evidenced by nitrotyrosine immunoreactivity in the transplanted hearts with LVH, was significantly increased.

From the clinical point of view, the aim of this study was to further elucidate the feasibility of optimal usage of "marginal" grafts in heart transplantation to identify and open-up new "druggable" therapeutic targets to increase the pool of donor organs.

In conclusion, alterations in donor hearts, as a consequence of hypertension and LVH, were not associated with LV graft dysfunction in the early phase of reperfusion after transplantation. Alterations in the expression of antioxidant enzyme genes (dual oxidase-1, serine peptidase inhibitor $b 1 b$, and sulfiredoxin-1) and the inflammatory cytokine $c c l 11$, and decreased acute inflammatory response may, in part, explain the tolerance of donor hearts with LVH to ischemia/reperfusion injury during heart transplantation. It remains unclear whether other essential pathways may also take part in this effect. Further studies are needed to evaluate whether LVH in donors regresses in the late post-transplant period.

\section{Study limitations}

First, our data suggests, without providing proof-ofconcept, that the alteration of genes, including dual oxidase-1, serine peptidase inhibitor b1b, sulfiredoxin1 and ccll1 may be possible targeting mechanisms when donor hearts with LVH are used. Second, in a clinical scenario, the use of hearts with LVH will be determined after careful selection, whereas in the present study LVH was left untreated. Finally, the possible adverse effects of immune cell activation, that can be triggered by heart transplantation, were not examined.

Supplementary Information The online version contains supplementary material available at https://doi.org/10.1007/s11357021-00348-8.

Acknowledgements This study was supported by the Deutsche Stiftung für Herzforschung (German Heart Research Foundation) (to S. Korkmaz-Icöz), by the Land Baden-Württemberg, Germany, and by the Medical Faculty of the University of Heidelberg, Germany (to Dr. S. Korkmaz-Icöz). The expert technical assistance of Karin Sonnenberg, Lutz Hoffmann, Tobias Mayer, and Patricia Kraft is gratefully acknowledged.

Author contribution Participated in research design: SKI, GS Participated in the writing of the paper: SKI, DA, SLo, PB, MR, AAS, MB, TR, GS

Participated in the performance of the research: SKI, DA, SL Contributed new reagents or analytic tools: SKI, MK, GS

Participated in data analysis: SKI, DA, SL, MB

Funding Open Access funding enabled and organized by Projekt DEAL. This study was supported by the Deutsche Stiftung für Herzforschung (German Heart Research foundation) (to Dr. S. Korkmaz-Icöz), by the Land Baden-Württemberg, Germany, by the Medical Faculty of the University of Heidelberg, Germany (to Dr. S. Korkmaz-Icöz), by the grant NVKP_16-1-2016-0017 (National Heart Program) with the support provided from the National Research, Development and Innovation Fund of Hungary (under the NVKP 16 funding scheme), and by the higher Education Institutional Excellence Program of the Ministry for Innovation and Technology in Hungary, within the framework of the Therapeutic Development thematic programs of the Semmelweis University.

\section{Declarations}

Conflict of interest The authors declare that they have no conflict of interest.

Open Access This article is licensed under a Creative Commons Attribution 4.0 International License, which permits use, sharing, adaptation, distribution and reproduction in any medium or format, as long as you give appropriate credit to the original author(s) and the source, provide a link to the Creative Commons licence, and indicate if changes were made. The images or other third party material in this article are included in the article's Creative Commons licence, unless indicated otherwise in a credit line to the material. If material is not included in the article's Creative Commons licence and your intended use is not permitted by statutory regulation or exceeds the permitted use, you will need to obtain permission directly from the copyright holder. To view a copy of this licence, visit http://creativecommons.org/licenses/by/4.0/. 


\section{References}

1. Aziz S, Soine LA, Lewis SL, Kruse AP, Allen MD, Levy WC, et al. Donor left ventricular hypertrophy increases risk for early graft failure. Transpl Int. 1997;10:446-50.

2. Cingolani OH, Yang XP, Cavasin MA, Carretero OA. Increased systolic performance with diastolic dysfunction in adult spontaneously hypertensive rats. Hypertension. 2003;41: 249-54.

3. Condorelli G, Morisco C, Stassi G, Notte A, Farina F, Sgaramella G, et al. Increased cardiomyocyte apoptosis and changes in proapoptotic and antiapoptotic genes bax and bcl-2 during left ventricular adaptations to chronic pressure overload in the rat. Circulation. 1999;99:3071-8.

4. Devereux RB, Alonso DR, Lutas EM, Gottlieb GJ, Campo E, Sachs I, et al. Echocardiographic assessment of left ventricular hypertrophy: comparison to necropsy findings. Am J Cardiol. 1986;57:450-8.

5. Felker GM, Milano CA, Yager JE, et al. Outcomes with an alternate list strategy for heart transplantation. J Heart Lung Transplant. 2005;24:1781-6.

6. Frey N, Olson EN. Cardiac hypertrophy: the good, the bad, and the ugly. Annu Rev Physiol. 2003;65:45-79.

7. Go AS, Mozaffarian D, Roger VL, Benjamin EJ, Berry JD, Borden WB, et al. Heart disease and stroke statistics-2013 update: a report from the American Heart Association. Circulation. 2013;127:e6-e245.

8. Goland S, Czer LS, Kass RM, et al. Use of cardiac allografts with mild and moderate left ventricular hypertrophy can be safely used in heart transplantation to expand the donor pool. J Am Coll Cardiol. 2008;51:1214-20.

9. Ikeda S, Hamada M, Hiwada K. Contribution of noncardiomyocyte apoptosis to cardiac remodelling that occurs in the transition from compensated hypertrophy to heart failure in spontaneously hypertensive rats. Clin Sci (Lond). 1999;97:239-46.

10. Kass DA, Beyar R, Lankford E, Heard M, Maughan WL, Sagawa K. Influence of contractile state on curvilinearity of in situ end-systolic pressure-volume relations. Circulation. 1989;79:167-78.

11. Korkmaz-Icoz S, Lehner A, Li S, et al. Left ventricular pressure-volume measurements and myocardial gene expression profile in type 2 diabetic Goto-Kakizaki rats. Am J Physiol Heart Circ Physiol. 2016;311:H958-71.

12. Korkmaz-Icoz S, Li S, Loganathan S, et al. Impairment of the Akt pathway in transplanted Type 1 diabetic hearts is associated with post-transplant graft injury. Interact Cardiovasc Thorac Surg. 2018.

13. Li S, Korkmaz S, Loganathan S, Weymann A, Radovits T, Barnucz E, et al. Acute ethanol exposure increases the susceptibility of the donor hearts to ischemia/reperfusion injury after transplantation in rats. PLoS One. 2012;7:e49237.

14. Li H, Pei F, Shao L, Chen J, Sun K, Zhang X, et al. Prevalence and risk factors of abnormal left ventricular geometrical patterns in untreated hypertensive patients. BMC Cardiovasc Disord. 2014a;14:136.

15. Li S, Loganathan S, Korkmaz S, et al. Transplantation of donor hearts after circulatory or brain death in a rat model. J Surg Res. 2014b;195:315-24.
16. Loganathan S, Korkmaz-Icoz S, Radovits T, et al. Effects of soluble guanylate cyclase activation on heart transplantation in a rat model. J Heart Lung Transplant. 2015;34:1346-53.

17. Lund LH, Edwards LB, Dipchand AI, Goldfarb S, Kucheryavaya AY, Levvey BJ, et al. The Registry of the International Society for Heart and Lung Transplantation: Thirty-third Adult Heart Transplantation Report-2016; Focus Theme: Primary Diagnostic Indications for Transplant. J Heart Lung Transplant. 2016;35:1158-69.

18. Luscher MS, Thygesen K, Ravkilde J, Heickendorff L. Applicability of cardiac troponin $\mathrm{T}$ and I for early risk stratification in unstable coronary artery disease. TRIM Study Group. Thrombin Inhibition in Myocardial ischemia. Circulation. 1997;96:2578-85.

19. Marelli D, Laks H, Fazio D, Moore S, Moriguchi J, Kobashigawa J. The use of donor hearts with left ventricular hypertrophy. J Heart Lung Transplant. 2000;19:496-503.

20. Maulik SK, Kumar S. Oxidative stress and cardiac hypertrophy: a review. Toxicol Mech Methods. 2012;22:359-66.

21. Mozaffarian D, Benjamin EJ, Go AS, Arnett DK, Blaha MJ, Cushman M, et al. Heart disease and stroke statistics-2015 update: a report from the American Heart Association. Circulation. 2015;131:e29-322.

22. Nicoletti A, Michel JB. Cardiac fibrosis and inflammation: interaction with hemodynamic and hormonal factors. Cardiovasc Res. 1999;41:532-43.

23. Okamoto K. Establishment of the stroke-prone spontaneously hypertensive rat (SHR). Circ Res. 1974;34:143-53.

24. Pacher P, Nagayama T, Mukhopadhyay P, Batkai S, Kass DA. Measurement of cardiac function using pressure-volume conductance catheter technique in mice and rats. Nat Protoc. 2008;3: 1422-34.

25. Pombo JF, Troy BL, Russell RO Jr. Left ventricular volumes and ejection fraction by echocardiography. Circulation. 1971;43:480-90.

26. Ravkilde J, Nissen H, Horder M, Thygesen K. Independent prognostic value of serum creatine kinase isoenzyme MB mass, cardiac troponin $\mathrm{T}$ and myosin light chain levels in suspected acute myocardial infarction. Analysis of 28 months of follow-up in 196 patients. J Am Coll Cardiol. 1995;25:574-81.

27. Sarvari SI, Gjesdal O, Gude E, Arora S, Andreassen AK, Gullestad L, et al. Early postoperative left ventricular function by echocardiographic strain is a predictor of 1-year mortality in heart transplant recipients. J Am Soc Echocardiogr. 2012;25:1007-14.

28. Schofield ZV, Woodruff TM, Halai R, Wu MC, Cooper MA. Neutrophils-a key component of ischemiareperfusion injury. Shock. 2013;40:463-70.

29. Snoeckx LH, van der Vusse GJ, Coumans WA, Willemsen $\mathrm{PH}$, Reneman RS. Differences in ischaemia tolerance between hypertrophied hearts of adult and aged spontaneously hypertensive rats. Cardiovasc Res. 1993;27:874-81.

30. Tan JL, Thakur K. Systolic Hypertension. In: StatPearls. Treasure Island; 2020.

31. Wever Pinzon O, Stoddard G, Drakos SG, Gilbert EM, Nativi JN, Budge D, et al. Impact of donor left ventricular hypertrophy on survival after heart transplant. Am J Transplant. 2011;11:2755-61.

32. Wilhelm MJ. Long-term outcome following heart transplantation: current perspective. J Thorac Dis. 2015;7:549-51. 
33. Zhang J, He Z, Guo J, Li Z, Wang X, Yang C, et al. Sulfiredoxin-1 protects against simulated ischaemia/ reperfusion injury in cardiomyocyte by inhibiting $\mathrm{PI} 3 \mathrm{~K} /$ AKT-regulated mitochondrial apoptotic pathways. Biosci Rep. 2016;36.

34. Zweifel M, Matozan K, Dahinden C, Schaffner T, Mohacsi P. Eotaxin/CCL11 levels correlate with myocardial fibrosis and mast cell density in native and transplanted rat hearts. Transplant Proc. 2010;42:2763-6.

Publisher's note Springer Nature remains neutral with regard to jurisdictional claims in published maps and institutional affiliations. 